\title{
The Data Acquisition Experiment Device by Parallel SPP Model Design
}

\author{
Chang Liu \\ Zaozhuang Vocational College of Science \& Technology, Zaozhuang, Shandong 277599, China
}

Keywords: Computer Practice, Visual C++, Data Acquisition, Standard Parallel Port (SPP).

\begin{abstract}
Using parallel SPP mode switch to input and output experiment device can simulate the collected data, and according to the principle of data acquisition program, data can be acquired for storage, query processing, output, etc. The simulation data can be used as environmental temperature, humidity, pressure and individual traits such as a variety of experimental data of a certain parameter values or attribute value. Independent research and development of experimental apparatus for curriculum design is as follows: for instance, expansion form C language demonstration to Visual C + + language, after simulation data collected by deepening the reform of computer learning.
\end{abstract}

\section{Introduction}

The current domestic and foreign application main goal is that return the practice of teaching reform, adapt to the need of the industry status and development [1] in the information age, pay attention to open to the students' participation, guidance, teaching process and cooperation incentive to cultivate the students' learning autonomy, optional sex and compound. University in our country is the developing trend of the research university, to cultivate students can attach importance to practice, to practice, fall in love with practical attitude, make full interest in using of the demonstration example, actively looking for opportunities, further encourage students want to do more, put the idea into practice, make innovation spirit rise to innovation [2]. Master the operation of characteristic of computational thinking, guide to put forward new problems.

\section{Technical background}

Standard configuration of existing computer parallel ports has three port registers of eight bit, including the data registers, the status registers and the control registers, the computer can read and write by the register to access the data of the parallel port. In many cases directly using the existing computer complete data input and output port is not impossible to achieve, must pass a certain conversion device to achieve. The utility model to solve the technical problem is to provide a data acquisition experiment device, the use of the device can realize parallel SPP model switch input and output [3].

\subsection{The technical scheme of the utility model.}

The device including rectangular shell, shell on the top with the order for D7 $\sim$ D0 just eight location identification. Below the location identification is equipped with upper and lower two rows and the location identification should be relatively light, ranked as the output light, discharge to the input light. Under two rows of lights for the eight data input KD series switches, KD series switch settings are respectively with the D7 D0 location identification should be relatively, each KD series switch is equipped with two switch input state 1 or 0 . On the sidewalls of the shell has a connection port and the device's start switch.

As shown in figure 1, the device including rectangular shell logo1 is on the top with the order for D7 D0 eight positioning logo 2. Below the target logo 2 is equipped with upper and lower two rows and the target logo 2 corresponding indicator light, for the output light is logo3, on the bottom of input light is logo 4. Under two rows of lights for the eight data input KD series switch is logo 5, KD series switch settings are respectively corresponding to the D7 D0 positioning logo 2, each logo $5 \mathrm{KD}$ 
series switch is equipped with two switch input state 1 or 0 . When a state of KD series switch to 1 , the above input indicator; When the series $\mathrm{KD}$, the state of the switch for 0 , above the input light is not bright. On the sidewalls of the shell 1 is equipped with the terminals logo 6 and start switch logo 7 of the device. when $\mathrm{Kn}(\mathrm{n}=0, \ldots, 7)$ input 1 , Dn location input indicator; Otherwise Dn position light is put out. OUT LED lights for the output data, works the same as the input data indicator.

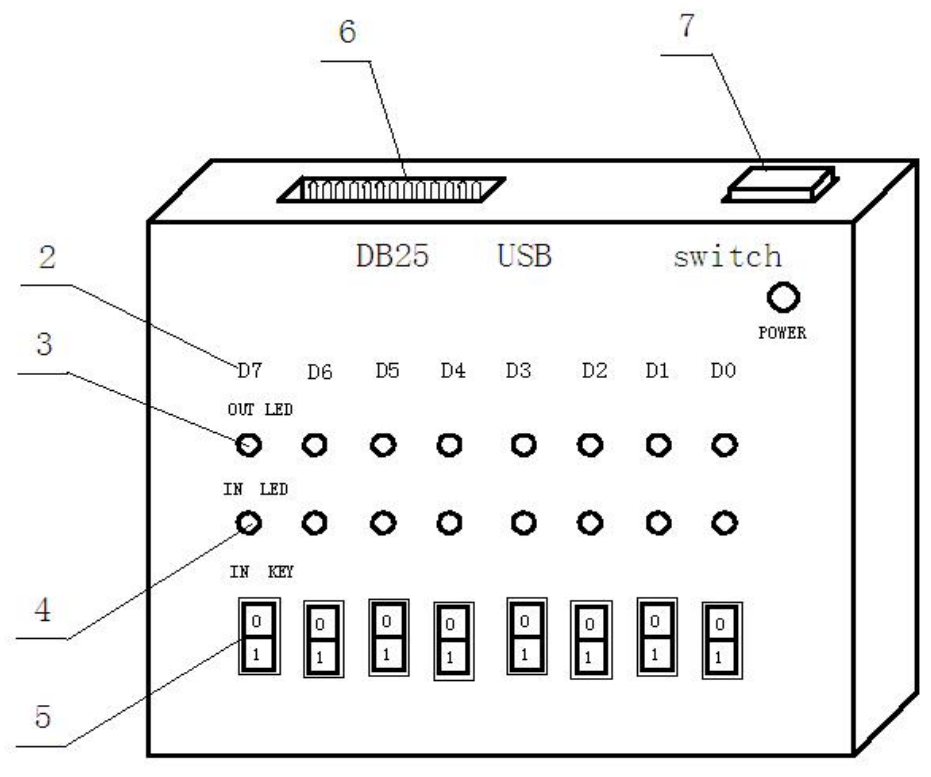

Fig. 1 SPP model switch input and output

\subsection{The principle of the utility model.}

The experimental system in the standard parallel port of computer SPP mode, using the data port D0 D7, a total of 8 bits for data output from the computer to the experiment device; Using control port C0 C3, a total of four control and state port (S3 S64 state a splicing into eight bits (C0 C3 for high four, S3 $\sim \mathrm{S} 6$ for low 4 bit) implement the data from experiment device to the computer's input.

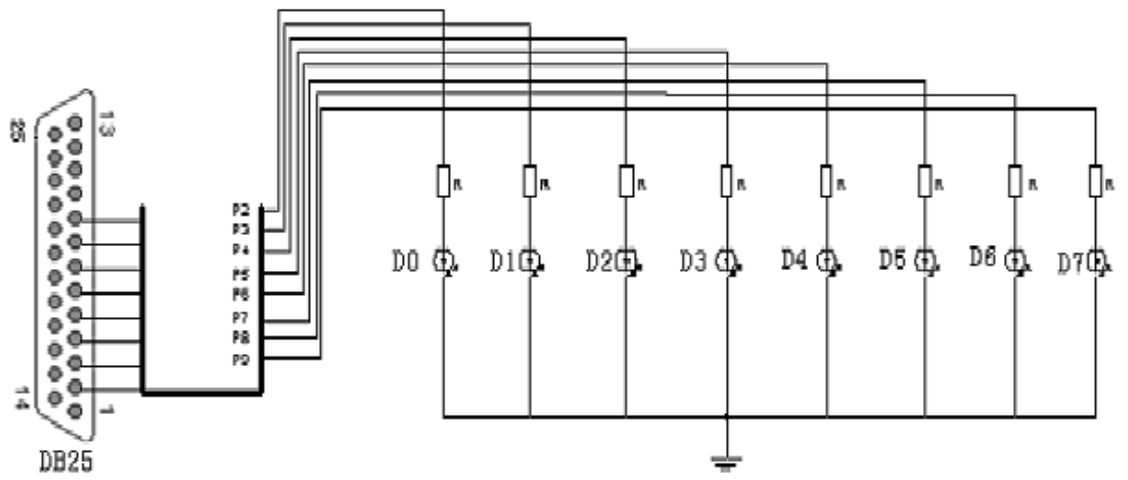

Fig. 2 SPP model’s principle

As shown in figure 2, OUT0 OUT7 for experiment data output pin; IN0 $\sim$ IN7 the data of experimental device input pin. On-off signal data from experiment device IN0 IN7 pin standard by computer parallel port connector pins as P15, P13, P12, P10, P1, P14, P16 and P17 into computer standard parallel port status register S3 S6 and control registers C0 C3. By programming control and status registers can be read and spliced into 1 byte of eight bit in the data. Then, the data will be joining together after processing the data through the program written data registers D0 $\sim \mathrm{D} 7$, this data by the computer standard parallel port connector pins P2 P9 was introduced into experimental apparatus OUT0 $\sim$ OUT7 pins, so as to realize the data from experiment device of computer parallel 
port to standard output.

\section{Using Installation Instructions and Method}

First, start the computer, enter the Windows environment, if you use the object-oriented programming languages such as Visual $\mathrm{C}++$, Visual VB ect; If you use the TC process oriented programming language, should better enter the Dos environment. Then, connect the DB25 end of the experiment device with the computer standard parallel port connection; Connect the USB port and the computer's USB port; Open experiment device the Power Switch, the Switch again to check the Power indicator light (Power), if the indicator, indicates the experimental device can work normally. At this time, you can start using the experiment device.

For example, the use of K0 $\sim$ K7 KD switch to collect data such as 23 degrees (corresponding the binary number as 00010111), press the corresponding KD switch place K4, K1, K2, K0 is 1, place K7, K6, K5, K3 is 0; Make the light D4, D2 and D0 input bright, implement the data input; Operate the programme to write data standard parallel port of computer data as 23, make the output of OUT LED correspond indicator D4, D2 and D0, so as to realize the data output. Parallel port connector with a total of 25 pins (the interface appearance is shown in figure 3).

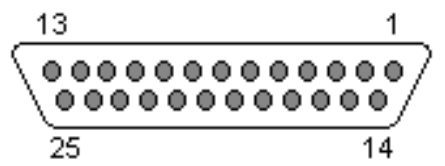

Fig. 3 standard parallel port 25 pin type D socket

Data register: data register is also called the data port. acquiesce in address as $378 \mathrm{H}$, its definition as shown in table 1.

Table 1 Data port pin definition

\begin{tabular}{ccccc}
\hline Register tag & Connector pins & Signal & Signal source & Inverter connector \\
\hline D0 & P2 & Data0 & PC & No \\
D1 & P3 & Data1 & PC & No \\
D2 & P4 & Data2 & PC & No \\
D3 & P5 & Data3 & PC & No \\
D4 & P6 & Data4 & PC & No \\
D5 & P7 & Data5 & PC & No \\
D6 & P8 & Data6 & PC & No \\
D7 & P9 & Data7 & PC & No
\end{tabular}

Status register: status register is also called the state port, the default address as $379 \mathrm{H}$, its definition as shown in table 2.

Table 2 State port pin definition

\begin{tabular}{ccccc}
\hline Register tag & Connector pins & Signal & Signal source & Inverter connector \\
\hline S0 & Time-Out & & \\
S1 & & unused & \\
S2 & unused & & \\
S3 & P15 & nError(nFault) & peripherals & No \\
S4 & P13 & Select & peripherals & No \\
S5 & P12 & PaperEnd & peripherals & No \\
S6 & P10 & nAck & peripherals & No \\
S7 & P11 & Busy & peripherals & Yes \\
\hline
\end{tabular}


Control register: control register is also called the console port, the default address as 37AH, its definition as shown in table 3.

Table 3 Control port pin definition

\begin{tabular}{ccccc}
\hline Register tag & Connector pins & Signal & $\begin{array}{c}\text { Signal } \\
\text { source }\end{array}$ & Inverter connector \\
\hline C0 & P1 & nStrobe & PC & Yes \\
C1 & P14 & nAutoLF & PC & Yes \\
C2 & P16 & nInit & PC & No \\
C3 & P17 & nSelectIn & PC & Yes \\
C4 & & IRQ & & \\
C5 & & unused & & \\
C6 & unused & \\
C7 & unused & \\
\hline
\end{tabular}

This experiment device for the student can be extended to real life related to the design of the topic, formulate feasible development course design plan, review the related textbooks and reference books, such as

(1) Determine the topic, set up the design team, make the requirement analysis (0.5 days);

(2) Internet access to information, analyze problems, formulate solutions (1 day);

(3) Program and debug (3 days);

(4) Submit design content and improvement (1 day).

Automation major, after the early stage of learning $\mathrm{c}++$ language was very interested in the experiment, the group on the basis of the original TurboC, using Visual $c++6.0$ to write the performance measurement, output, sorting, and query information, and through the file record measurement information and summary. Divided into a responsible: measurement module (innew () function); Data storage module; Join together of each module; Data input module; Data statistics module; Output module. Analyzed by the List class innew () function is complete, the function calls the Winio library function: GetPortVal (0 x379, \& getval, 1); CTRLG x37a SetPortVal (0, 1); A friend function using the class List, private members of the List.

\section{Conclusions}

Based on increased course in all kinds of professional curriculum design process, in order to can reach the practical exploration or project for the outline of the topic, so that the students in the design process has a clear train of thought, through the practice data feedback to prove the study effect is good, eventually to foster and train students' ability of autonomous learning.

(1) Due to previous experimental verification, more students have no development design thought and ability.

(2) In shorter design time, improve the practice teaching of practical methods, to strengthen and perfect the practice teaching link of the teaching effect.

\section{References}

[1] Nine Schools League (C9): Journal of China university teaching, (2010) No.9, p.4. (In Chinese)

[2] Sanz, D; Ruiz, M; Castro, R; Vega, J; Lopez, J. M; Barrera, E;Utzel, N : IEEE Transactions on Nuclear Science, ISSN 0018-9499, (2013)No 5, p. 3446 - 3453.

[3] S.X. Chen. A Data Acquisition Experiment Device :China Patent CN203644038U. (2014). 